\title{
Independence of algebraic monodromy groups in compatible systems
}

\author{
Federico Amadio Guidi ${ }^{1}$
}

Accepted: 2 May 2021 / Published online: 6 July 2021

(C) The Author(s) 2021

\begin{abstract}
In this paper we develop a general method to prove independence of algebraic monodromy groups in compatible systems of representations, and we apply it to deduce independence results for compatible systems both in automorphic and in positive characteristic settings. In the abstract case, we prove an independence result for compatible systems of Lie-irreducible representations, from which we deduce an independence result for compatible systems admitting what we call a Lie-irreducible decomposition. In the case of geometric compatible systems of Galois representations arising from certain classes of automorphic forms, we prove the existence of a Lie-irreducible decomposition. From this we deduce an independence result. We conclude with the case of compatible systems of Galois representations over global function fields, for which we prove the existence of a Lie-irreducible decomposition, and we deduce an independence result. From this we also deduce an independence result for compatible systems of lisse sheaves on normal varieties over finite fields.
\end{abstract}

Keywords Compatible systems of Galois representations · Independence of algebraic monodromy groups $\cdot$ Automorphic compatible systems $\cdot$ Compatible systems over global function fields

Mathematics Subject Classification 11F80 - 11F70 - 11S37 · 14G17

\section{Introduction}

Questions on $\ell$-independence of algebraic monodromy groups in compatible systems originate in the literature from a classical result by Serre, see [19], which states that if $E$ is an elliptic curve without complex multiplication over a number field, then the image of the Galois representation on the $\ell$-adic Tate module of $E$ is an open subgroup

Federico Amadio Guidi

federico.amadio@maths.ox.ac.uk

1 Mathematical Institute, University of Oxford, Oxford, UK

Birkhäuser 
of $\mathrm{GL}_{2}\left(\mathbb{Z}_{\ell}\right)$ for every prime $\ell$, and is equal to $\mathrm{GL}_{2}\left(\mathbb{Z}_{\ell}\right)$ for all but finitely many primes $\ell$. The problem of extending this result to more general geometric settings led, for instance, to the formulation of the Mumford-Tate conjecture for abelian varieties, see [16]. Nevertheless, one can naturally study $\ell$-independence questions for any abstract rational compatible system of representations. In this context, the foundational work of Larsen and Pink, see [15], provides some breakthroughs.

It is immediate to notice how the main results of [15] can be extended verbatim to compatible systems having coefficients in any number field. In our paper, we use these results to develop a general method to prove $\lambda$-independence of the neutral components of the algebraic monodromy groups of compatible systems over a finite extension of the field of coefficients. The main idea is that one can prove a $\lambda$-independence result of this form whenever a compatible system admits a Lie-irreducible decomposition over a finite extension of its field of coefficients. Let us briefly sketch our approach. First of all, we apply the results of [15] to prove $\lambda$-independence of the neutral components of the algebraic monodromy groups of compatible systems of Lie-irreducible (i.e. irreducible after restricting to any open subgroup) representations over a finite extension of the field of coefficients, see Proposition 4.1. We then introduce the notion of Lieirreducible decomposition, see Definition 4.2, and we deduce from the Lie-irreducible case an analogous $\lambda$-independence result for compatible systems admitting a Lieirreducible decomposition, see Corollary 4.4.

In the case of automorphic compatible systems (and actually for a slightly larger class of geometric compatible systems), we prove the existence of a Lie-irreducible decomposition at a set of primes of positive Dirichlet density, see Theorem 5.4, from which we deduce a $\lambda$-independence result, see Corollary 5.5. Assuming a classical irreducibility conjecture for automorphic compatible systems, the set of primes of positive Dirichlet density can be actually replaced by a set of primes of Dirichlet density 1 .

In the positive characteristic setting, we prove the existence of a Lie-irreducible decomposition for compatible systems of representations of the absolute Galois group of a global function field, see Theorem 6.2, from which we deduce a $\lambda$-independence result, see Corollary 6.3. We also deduce a $\lambda$-independence result for compatible systems of lisse sheaves on normal varieties over finite fields, see Corollary 6.5.

The structure of this paper is the following. In Sect. 1 we introduce the basic terminology, while in Sects. 2 and 3 we recall the main tools from [15] which will be useful in Sect. 4. The general method of this paper and the main results in the general setting are presented in Sect. 4. Finally, we see applications of the results of Sect. 4 to the case of geometric compatible systems of Galois representations in Sect. 5, and to compatible systems in the positive characteristic case in Sect. 6.

\section{Notation}

For a prime $\ell$, we denote by $\zeta_{\ell}$ a primitive $\ell$ th-root of 1 .

Given a profinite group $\Gamma$, an integer $n \geq 1$, and a continuous representation $\rho: \Gamma \rightarrow \mathrm{GL}_{n}\left(\overline{\mathbb{Q}}_{\ell}\right)$, we let $\bar{\rho}: \Gamma \rightarrow \mathrm{GL}_{n}\left(\overline{\mathbb{F}}_{\ell}\right)$ denote the semisimplification of the reduction of $\rho$ modulo $\ell$, which is defined up to conjugacy. 
Given a field $k$, we let $\bar{k}$ be an algebraic closure of $k$, and $k^{\mathrm{s}}$ be a separable closure of $k$ inside $\bar{k}$, and we denote by $\Gamma_{k}=\operatorname{Gal}\left(k^{\mathrm{s}} / k\right)$ the absolute Galois group of $k$.

Given a global field $F$, we denote by $|F|$ the set of finite places of $F$. If $\Sigma$ is a set of places of $F$, and $\ell$ and $p$ are primes, we set $\Sigma_{\ell}=\{v \in \Sigma: v \mid \ell\}$ and $\Sigma_{\neq p}=\cup_{\ell^{\prime} \neq p} \Sigma_{\ell^{\prime}}$. We denote by $\mathbb{A}_{F}$ the ring of adeles of $F$.

If $K$ is a non-Archimedean local field, we let $I_{K}$ be the inertia subgroup of $\Gamma_{K}$, and Frob $_{K} \in \Gamma_{K} / I_{K}$ be the geometric Frobenius. When $K=F_{v}$, for $F$ a global field, and $v$ a finite place of $F$, we write Frob $_{v}=$ Frob $_{F_{v}}$.

If $K$ is a finite extension of $\mathbb{Q}_{p}$, given a Weil-Deligne representation $(r, N)$ of the Weil group $W_{K}$ of $K$, we let $(r, N)^{\mathrm{F}-\text { ss }}$ denote its Frobenius-semisimplification. Given a continuous representation $\rho: \Gamma_{K} \rightarrow \mathrm{GL}_{n}\left(\overline{\mathbb{Q}}_{\ell}\right)$, with $\ell \neq p$, we denote by $\mathrm{WD}(\rho)$ the corresponding Weil-Deligne representation of $W_{K}$. We denote by $\operatorname{rec}_{K}$ the local Langlands correspondence for $\mathrm{GL}_{n}$ over $K$ of [11]. When $K=F_{v}$, for $F$ a number field, and $v$ a finite place of $F$, we write $\operatorname{rec}_{v}=\operatorname{rec}_{F_{v}}$.

If $K$ is a finite extension of $\mathbb{Q}_{\ell}$, if $V$ is a finite dimensional vector space over $\overline{\mathbb{Q}}_{\ell}$, and $\rho: \Gamma_{K} \rightarrow \mathrm{GL}(V)$ is a continuous semisimple de Rham representation, we denote by $\operatorname{WD}(\rho)$ the corresponding Weil-Deligne representation of $W_{K}$. Also, given an embedding $\tau: K \rightarrow \overline{\mathbb{Q}}_{\ell}$ we define the multiset $\operatorname{HT}_{\tau}(\rho)$ of $\tau$-Hodge-Tate weights of $\rho$ to be the multiset of $\operatorname{dim}_{\overline{\mathbb{Q}}_{\ell}} V$ integers containing $i$ with multiplicity $\operatorname{dim}_{\overline{\mathbb{Q}}_{\ell}}\left(V \otimes_{\tau, K} \widehat{\bar{K}}(i)\right)^{\Gamma_{F}}$, where $\widehat{\bar{K}}$ denotes the completion of an algebraic closure of $K$. If $F$ is a number field, if $V$ is a finite dimensional vector space over $\overline{\mathbb{Q}}_{\ell}$, and $\rho: \Gamma_{F} \rightarrow \mathrm{GL}(V)$ is a continuous semisimple representation which is de Rham at all places above $\ell$, given an embedding $\tau: F \rightarrow \overline{\mathbb{Q}}_{\ell}$ we define the multiset of $\tau$ Hodge-Tate weights of $\rho$ to be $\operatorname{HT}_{\tau}(\rho)=\operatorname{HT}_{\tau}\left(\left.\rho\right|_{\Gamma_{F_{v(\tau)}}}\right)$, where $v(\tau)$ is the place of $F$ induced by $\tau$.

Given a scheme $X$ of finite type over $\mathbb{F}_{q}$, where $q$ is a power of $p$, we denote by $|X|$ its set of closed points. For each $x \in|X|$, let $\bar{x}$ be an algebraic geometric point of $X$ localised at $x$, and let $\operatorname{Frob}_{x} \in \Gamma_{k(x)}=\operatorname{Gal}(k(\bar{x}) / k(x))$ be the geometric Frobenius. Given a sheaf $\mathcal{F}$ on $X$, we write $\operatorname{det}\left(1-\operatorname{Frob}_{x} t, \mathcal{F}\right)$ for the characteristic polynomial of the image of Frob $_{x}$ under the monodromy representation of $\Gamma_{k(x)}$ on $\mathcal{F}_{k(\bar{x})}$.

\section{Compatible systems and algebraic monodromy groups}

A group with Frobenii, or simply an $F$-group, is a pair $\left(\Gamma,\left\{F_{\alpha}\right\}_{\alpha \in A}\right)$ where $\Gamma$ is a profinite group, and $\left\{F_{\alpha}\right\}_{\alpha \in A}$ is a collection of elements of $\Gamma$, called Frobenius elements, which are dense in $\Gamma$. Motivating examples for the definition of group with Frobenii are clearly the absolute Galois group of a global field, together with the collection of geometric Frobenii at its finite places, and the étale fundamental group of a scheme of finite type over a finite field, together with the collection of geometric Frobenii at its closed points.

Let $\left(\Gamma,\left\{F_{\alpha}\right\}_{\alpha \in A}\right)$ be a group with Frobenii, let $E$ be a number field, and let $\Lambda$ be a set of finite places of $E$. We give the following definition. ${ }^{1}$

$\overline{1}$ Compare [15, Definition 6.5]. 
Definition 1.1 A compatible system of rank $n$ representations of $\Gamma$ defined over $E$ is a family $\mathcal{R}=\left\{\rho_{\lambda}\right\}_{\lambda \in \Lambda}$ of continuous representations

$$
\rho_{\lambda}: \Gamma \rightarrow \mathrm{GL}_{n}\left(\bar{E}_{\lambda}\right)
$$

such that there is a subset $\mathcal{X} \subset A \times \Lambda$ satisfying the following conditions.

(1) For every $\alpha \in A,(\alpha, \lambda) \in \mathcal{X}$ for all but finitely many $\lambda \in \Lambda$.

(2) For all $(\alpha, \lambda) \in \mathcal{X}$, the characteristic polynomial of $\rho_{\lambda}\left(F_{\alpha}\right)$ has coefficients in $E$ and is independent of $\lambda$.

(3) For any places $\lambda_{1}, \ldots, \lambda_{m} \in \Lambda$, the set $\left\{F_{\alpha}:\left(\alpha, \lambda_{i}\right) \in \mathcal{X}\right.$ for all $\left.i=1, \ldots, m\right\}$ is dense in $\Gamma$.

In the last two sections we will see two main (motivating) classes of examples of compatible systems, i.e. geometric compatible systems of Galois representations, see Sect. 5, and compatible systems of lisse sheaves on schemes of finite type over finite fields, see Sect. 6.

We say that a compatible system $\mathcal{R}=\left\{\rho_{\lambda}\right\}_{\lambda \in \Lambda}$ of rank $n$ representations of $\Gamma$ defined over $E$ has coefficients in a finite extension $E^{\prime}$ of $E$ if for any $\lambda \in \Lambda$ the image of $\rho_{\lambda}$ is contained in $\mathrm{GL}_{n}\left(E_{\lambda^{\prime}}^{\prime}\right)$ for some place $\lambda^{\prime}$ of $E^{\prime}$ above $\lambda$.

Given two sets $\mathcal{L}$ and $\mathcal{L}^{\prime}$ of rational primes, and a non empty subset $\mathcal{L}^{\prime \prime} \subset \mathcal{L} \cap \mathcal{L}^{\prime}$, we say that two compatible systems $\mathcal{R}=\left\{\rho_{\lambda}\right\}_{\lambda \in \Lambda}$ and $\mathcal{R}^{\prime}=\left\{\rho_{\lambda}^{\prime}\right\}_{\lambda \in \Lambda^{\prime}}$ defined over a number field $E$, where $\Lambda=\cup_{\ell \in \mathcal{L}}|E|_{\ell}$ and $\Lambda^{\prime}=\cup_{\ell \in \mathcal{L}^{\prime}}|E|_{\ell}$, are isomorphic at $\mathcal{L}^{\prime \prime}$ if $\rho_{\lambda} \cong \rho_{\lambda}^{\prime}$ for all $\ell \in \mathcal{L}^{\prime \prime}$ and $\lambda \mid \ell$. In this case, we write $\mathcal{R} \cong\left(\mathcal{L}^{\prime \prime}\right) \mathcal{R}^{\prime}$. When $\mathcal{R}$ and $\mathcal{R}^{\prime}$ have coefficients in $E$, we say that they are isomorphic over $E$ if the isomorphism $\rho_{\lambda} \cong \rho_{\lambda}^{\prime}$ is defined over $E_{\lambda}$ for all $\ell \in \mathcal{L}^{\prime \prime}$ and $\lambda \mid \ell$.

Let $\left(\Gamma,\left\{F_{\alpha}\right\}_{\alpha \in A}\right)$ be a group with Frobenii, and let $\mathcal{R}=\left\{\rho_{\lambda}\right\}_{\lambda \in \Lambda}$ be a compatible system of rank $n$ representations of $\Gamma$ with coefficients in a number field $E$. For any $\lambda \in \Lambda$, denote by $G_{\lambda}$ the Zariski closure of $\rho_{\lambda}(\Gamma)$ in $\operatorname{GL}_{n}\left(E_{\lambda}\right)$. This is an algebraic subgroup of $\mathrm{GL}_{n}, E_{\lambda}$, which we call the algebraic monodromy group of $\rho_{\lambda}$. We recall the following lemma.

Lemma 1.2 [15, Lemma 6.9] If $\rho_{\lambda}$ is semisimple, then $G_{\lambda}$ is reductive. If $\rho_{\lambda}$ is absolutely irreducible, then $G_{\lambda}$ is reductive, and its natural representation $G_{\lambda} \rightarrow \mathrm{GL}_{n, E_{\lambda}}$ is absolutely irreducible.

Let $G_{\lambda}^{\circ}$ be the connected component of the identity of $G_{\lambda}$. It is immediate to notice that if $\Gamma^{\prime}$ is an open subgroup of $\Gamma$, and $G_{\lambda}^{\prime}$ denotes the Zariski closure of $\rho_{\lambda}\left(\Gamma^{\prime}\right)$ in $\mathrm{GL}_{n}\left(E_{\lambda}\right)$, then the connected component of the identity $G_{\lambda}^{\prime \circ}$ of $G_{\lambda}^{\prime}$ coincides with $G_{\lambda}^{\circ}$.

In the next sections, we consider the problem of studying the algebraic monodromy groups $G_{\lambda}$ of $\mathcal{R}=\left\{\rho_{\lambda}\right\}_{\lambda \in \Lambda}$ varying $\lambda$. The hope in this context is to find a global algebraic subgroup $G$ of $\mathrm{GL}_{n, E}$ defined over $E$ such that $G_{\lambda}$ is conjugate to $G \times{ }_{E} E_{\lambda}$ over $E_{\lambda}$ for any $\lambda \in \Lambda$. This is the prototype of a $\lambda$-independence problem for the compatible system $\mathcal{R}$. Unfortunately, proving a $\lambda$-independence result of this form in the abstract setting is actually too optimistic. For this reason, we will focus on weaker $\lambda$-independence statements. 


\section{The formal character and the variety of characteristic polynomials}

If $G$ is a reductive group over a field $k$ of characteristic zero, $T \subset G$ is a maximal torus of $G$ over $k$, and $\rho: G \rightarrow \mathrm{GL}_{n, k}$ is a representation of $G$ over $k$, we define the formal character of $\rho$ to be the restriction of $\rho$ to $T$.

Let ch : $\mathrm{GL}_{n, k} \rightarrow \mathbb{G}_{m, k} \times \mathbb{A}_{k}^{n-1}$ be the morphism which associates to a matrix the coefficients of its characteristic polynomial. Given a connected reductive algebraic subgroup $G$ of $\mathrm{GL}_{n, k}$, and a maximal torus $T \subset G$ over $k$, we have that $\operatorname{ch}(G)=\operatorname{ch}(T)$ is a variety defined over $\mathbb{Q}$, which determines uniquely the formal character of the natural representation $G \rightarrow \mathrm{GL}_{n, k}$ up to isomorphism, see [15, §4].

Let $\mathbb{G}_{m, k}^{n}$ be the split maximal torus of $\mathrm{GL}_{n, k}$, and let $T_{0} \subset \mathbb{G}_{m, k}^{n}$ be a subtorus such that $T_{0} \times_{k} \bar{k}$ is conjugate to $T \times_{k} \bar{k}$ over $\bar{k}$. Since the semisimple part of any point in $G$ can be conjugated into $T_{0}$ over $\bar{k}$, we have that $\operatorname{ch}(G)=\operatorname{ch}\left(T_{0}\right)$ pointwise. We have that the Weyl group Norm $\mathrm{GL}_{n, k}\left(\mathbb{G}_{m, k}^{n}\right) / \operatorname{Cent}_{\mathrm{GL}_{n, k}}\left(\mathbb{G}_{m, k}^{n}\right)$ of $\mathrm{GL}_{n, k}$ with respect to $\mathbb{G}_{m, k}^{n}$ is the symmetric group $\mathfrak{S}_{n}$, acting on $\mathbb{G}_{m, k}^{n}$ by permutation of factors. For every $\sigma \in \mathfrak{S}_{n} \backslash \operatorname{Cent}_{\mathfrak{S}_{n}}\left(T_{0}\right)$, we define a proper subgroup $H_{\sigma} \subset T_{0}$ in the following way. We let $H_{\sigma}=\{t \in T: \sigma(t)=t\}$ if $\sigma\left(T_{0}\right)=T_{0}$, and $H_{\sigma}=T_{0} \cap \sigma\left(T_{0}\right)$ otherwise. We define now $Y$ to be the union of all $\operatorname{ch}\left(H_{\sigma}\right)$. This is a Zariski-closed proper subset of $\operatorname{ch}\left(T_{0}\right)=\operatorname{ch}(G)$. We say that a point $g \in G$ is $\Gamma$-regular if $\operatorname{ch}(g) \notin Y$. We have the following result.

Proposition 2.1 [15, Proposition 4.7].

(1) For every $x \in(\operatorname{ch}(G) \backslash Y)(k)$, there exist a torus $T \subset \mathrm{GL}_{n, k}$, and an element $t \in T(k)$, such that $\operatorname{ch}(T)=\operatorname{ch}(G)$ and $\operatorname{ch}(t)=x$. The pair $(t, T)$ is unique up to conjugation by $\mathrm{GL}_{n}(k)$.

(2) Let $g \in G(k)$ be $\Gamma$-regular. Then $g$ lies in a unique maximal torus $T$ of $G$, and the $\mathrm{GL}_{n}(k)$-conjugacy class of $(g, T)$ in uniquely determined by $\operatorname{ch}(g)$ and $\operatorname{ch}(G)$.

Let $\left(\Gamma,\left\{F_{\alpha}\right\}_{\alpha \in A}\right)$ be a group with Frobenii, and let $\mathcal{R}=\left\{\rho_{\lambda}\right\}_{\lambda \in \Lambda}$ be a compatible system of rank $n$ semisimple representations of $\Gamma$ with coefficients in a number field $E$. Keep notations as in Sect. 1. We have:

Proposition 2.2 [15, Proposition 6.12] The formal character of the natural representation $G_{\lambda}^{\circ} \times_{E_{\lambda}} \bar{E}_{\lambda} \rightarrow \mathrm{GL}_{n, \bar{E}_{\lambda}}$ is independent of $\lambda$. More precisely, there exist a torus $T_{0}$ over $E$ and a faithful representation $\rho_{0}: T_{0} \rightarrow \mathrm{GL}_{n, E}$ such that $T_{0} \times_{E} \bar{E}_{\lambda}$ is isomorphic to a maximal torus in $G_{\lambda}^{\circ} \times E_{\lambda} \bar{E}_{\lambda}$, and $\rho_{0} \otimes_{E} \bar{E}_{\lambda}$ is equivalent to the formal character of $G_{\lambda}^{\circ} \times E_{\lambda} \bar{E}_{\lambda} \rightarrow \mathrm{GL}_{n, \bar{E}_{\lambda}}$ for all $\lambda \in \Lambda$.

Proposition 2.3 [15, Proposition 6.14] There is an open normal subgroup $\Gamma^{\prime} \subset \Gamma$ such that $\rho_{\lambda}$ induces an isomorphism $\Gamma / \Gamma^{\prime} \stackrel{\sim}{\rightarrow} G_{\lambda} / G_{\lambda}^{\circ}$ for all $\lambda \in \Lambda$.

Note that Proposition 2.3 implies that the group $G_{\lambda} / G_{\lambda}^{\circ}$ of connected components of $G_{\lambda}$ is independent of $\lambda$. Also, if we let $A^{\prime}=\left\{\alpha \in A: F_{\alpha} \in \Gamma^{\prime}\right\}$, then the pair $\left(\Gamma^{\prime},\left\{F_{\alpha}\right\}_{\alpha \in A^{\prime}}\right)$ is a group with Frobenii, and $\mathcal{R}^{\prime}=\left\{\rho_{\lambda}^{\prime}=\left.\rho_{\lambda}\right|_{\Gamma^{\prime}}\right\}_{\lambda \in \Lambda}$ forms a compatible system, with $\mathcal{X}^{\prime}=\mathcal{X} \cap\left(A^{\prime} \times \Lambda\right)$. Clearly, the algebraic monodromy group of $\rho_{\lambda}^{\prime}$ is $G_{\lambda}^{\circ}$ for every $\lambda \in \Lambda$. 


\section{Frobenius tori and the splitting field of a compatible system}

Let $\left(\Gamma,\left\{F_{\alpha}\right\}_{\alpha \in A}\right)$ be a group with Frobenii, and let $\mathcal{R}=\left\{\rho_{\lambda}\right\}_{\lambda \in \Lambda}$ be a compatible system of rank $n$ semisimple representations of $\Gamma$ with coefficients in a number field $E$. Let us assume in this section that for every $\lambda \in \Lambda$ the reductive group $G_{\lambda}$ is connected. We have the following result.

Proposition 3.1 [15, Proposition 7.2] For any $\lambda \in \Lambda$, the set of all $\gamma \in \Gamma$ such that $\rho_{\lambda}(\gamma)$ is $\Gamma$-regular is open and dense in $\Gamma$.

For every $(\alpha, \lambda) \in \mathcal{X}$, the point $\operatorname{ch}\left(\rho_{\lambda}\left(F_{\alpha}\right)\right) \in\left(\mathbb{G}_{m, E_{\lambda}} \times \mathbb{A}_{E_{\lambda}}^{n-1}\right)(E)$ depends only on $\alpha$, and so the condition on $\rho_{\lambda}\left(F_{\alpha}\right)$ being $\Gamma$-regular depends only on $\alpha$ as well. When this condition holds, we simply say that $\alpha$ is $\Gamma$-regular. By Proposition 3.1, if we replace $A$ with the set of $\Gamma$-regular $\alpha \in A$ the corresponding set $\mathcal{X} \subset A \times \Lambda$ still satisfies the conditions (1)-(3) in Definition 1.1. From now on, let us assume that every $\alpha \in A$ is $\Gamma$-regular.

By Proposition 2.1, for every $\alpha \in A$ we are then given a torus $T_{\alpha} \subset \mathrm{GL}_{n, E}$, canonical up to conjugacy, associated to $\operatorname{ch}\left(\rho_{\lambda}\left(F_{\alpha}\right)\right)$, for $\lambda \in \Lambda$ such that $(\alpha, \lambda) \in \mathcal{X}$, so that the set $\mathcal{X}$ satisfies the following conditions.

(1) For every $\alpha \in A,(\alpha, \lambda) \in \mathcal{X}$ for all but finitely many $\lambda \in \Lambda$.

(2) For all $(\alpha, \lambda) \in \mathcal{X}, T_{\alpha} \times_{E} E_{\lambda}$ is conjugate to a maximal torus of $G_{\lambda}$ over $E_{\lambda}$.

(3) For all pairwise distinct places $\lambda_{1}, \ldots, \lambda_{m} \in \Lambda$, and all maximal tori $T_{i}$ of $G_{\lambda_{i}}$, there exists an $\alpha \in A$ such that, for every $i,\left(\alpha, \lambda_{i}\right) \in \mathcal{X}$ and $T_{\alpha} \times_{E} E_{\lambda_{i}}$ is conjugate to $T_{i}$ over $E_{\lambda_{i}}$.

The tori $T_{\alpha}$ over $E$ are called Frobenius tori. For every $\alpha \in A$, let $L_{\alpha}$ be the splitting field of $T_{\alpha}$ over $E_{\alpha}$, that is the smallest degree extension $L_{\alpha} / E$ such that $T_{\alpha} \times_{E} L_{\alpha}$ is split. Let $L$ be the intersection of all $L_{\alpha}$. We call this field the splitting field of the compatible system $\mathcal{R}$.

Assume now that $\Lambda=\cup_{\ell \in \mathcal{L}}|E|_{\ell}$, where $\mathcal{L}$ is a set of rational primes of Dirichlet density $\sigma>0$. The role of Frobenius tori and the splitting field of a compatible system is clarified by the following results.

Proposition 3.2 [15, Proposition 8.9] There exists a subset $\mathcal{L}^{\prime}$ of $\mathcal{L}$ of Dirichlet density $\sigma$ such that for each $\ell \in \mathcal{L}^{\prime}$ and $\lambda \mid \ell$ the connected reductive group $G_{\lambda}$ is unramified, and split over $L E_{\lambda}$.

Corollary 3.3 Let $\mathcal{L}^{\prime}$ be a subset of $\mathcal{L}$ such that for each $\ell \in \mathcal{L}^{\prime}$ and $\lambda \mid \ell$ the group $G_{\lambda}$ is unramified. Then, there exist a $E$-split torus $T_{0}$ and a faithful representation $\rho_{0}: T_{0} \rightarrow \mathrm{GL}_{n, E}$ such that, for each $\ell \in \mathcal{L}^{\prime}$, each $\lambda \mid \ell$, and each finite place $\mathfrak{l}$ of $L$ lying above $\lambda$, the torus $T_{0} \times{ }_{E} L_{\mathfrak{l}}$ is conjugate to a maximal torus in $G_{\lambda} \times E_{\lambda} L_{\mathfrak{l}}$ over $L_{\mathfrak{l}}$, and $\rho_{0} \otimes_{E} L_{\mathfrak{l}}$ is equivalent to the formal character of $G_{\lambda} \times E_{\lambda} L_{\mathfrak{l}} \rightarrow \mathrm{GL}_{n, L_{\mathfrak{l}}}$ over $L_{\mathfrak{l}}$.

Theorem 3.4 (part of [15, Theorem 9.4]) Assume that each $\rho_{\lambda}$ is absolutely irreducible, and that $L=E$. Let $\mathcal{L}^{\prime}$ be a subset of $\mathcal{L}$ such that for each $\ell \in \mathcal{L}^{\prime}$ and $\lambda \mid \ell$ the group $G_{\lambda}$ is unramified. Then, for all $\ell \in \mathcal{L}^{\prime}$ and $\lambda \mid \ell$ the root datum $\Psi_{\lambda}$ of $G_{\lambda}$ is independent of $\lambda$ up to isomorphism. 


\section{Independence in compatible systems of Lie-irreducible representations}

Let $\left(\Gamma,\left\{F_{\alpha}\right\}_{\alpha \in A}\right)$ be a group with Frobenii, let $E$ be a number field, let $\mathcal{L}$ be a set of rational primes of Dirichlet density $\sigma>0$, and let $\Lambda=\cup_{\ell \in \mathcal{L}}|E|_{\ell}$. The following result holds.

Proposition 4.1 Let $\mathcal{R}=\left\{\rho_{\lambda}\right\}_{\lambda \in \Lambda}$ be a compatible system of rank $n$ absolutely Lieirreducible ${ }^{2}$ representations of $\Gamma$ with coefficients in $E$. Let $\mathcal{L}^{\prime}$ be a subset of $\mathcal{L}$ such that for each $\ell \in \mathcal{L}^{\prime}$ and $\lambda \mid \ell$ the group $G_{\lambda}^{\circ}$ is unramified. Then, there exist a finite extension $L$ of $E$ and a split connected reductive algebraic subgroup $G$ of $\mathrm{GL}_{n, L}$ defined over $L$ such that for each $\ell \in \mathcal{L}^{\prime}$, each $\lambda \mid \ell$, and each finite place $\mathfrak{l}$ of $L$ lying above $\lambda$, the group $G_{\lambda}^{\circ} \times E_{\lambda} L_{\mathfrak{l}}$ is conjugate to $G \times{ }_{L} L_{\mathfrak{l}}$ over $L_{\mathfrak{l}}$.

Proof Let $\Gamma^{\prime}$ be the open subgroup of $\Gamma$ given by Proposition 2.3. As each $\rho_{\lambda}$ is absolutely Lie-irreducible, the restrictions $\left.\rho_{\lambda}\right|_{\Gamma^{\prime}}$ are still absolutely irreducible. Up to restrict to $\Gamma^{\prime}$, we can assume each $G_{\lambda}$ to be connected. Let $L$ be the splitting field of $\mathcal{R}$, let $\mathfrak{L}^{\prime}=\cup_{\ell \in \mathcal{L}^{\prime}}|L|_{\ell}$, and for each $\mathfrak{l} \in \mathfrak{L}^{\prime}$, denoting by $\lambda$ its restriction to $E$, let $\rho_{\mathfrak{l}}=\rho_{\lambda} \otimes_{E_{\lambda}} L_{\mathfrak{l}}$. Consider now the compatible system $\left\{\rho_{\mathfrak{l}}\right\}_{\mathfrak{l} \in \mathfrak{L}^{\prime}}$. Clearly, the algebraic monodromy group $G_{\mathfrak{l}}$ of $\rho_{\mathfrak{l}}$ is equal to $G_{\lambda} \times{ }_{E_{\lambda}} L_{\mathfrak{l}}$ by construction. By Theorem 3.4, the root datum $\Psi_{\mathfrak{l}}$ of $G_{\mathfrak{l}}$ is independent of $\mathfrak{l}$ up to isomorphism. Fix such a root datum $\Psi$. Then, by [8, Theorem A.4.6], there exists a (unique up to isomorphism) split connected reductive subgroup $G$ of $\mathrm{GL}_{n, L}$ with root datum $\Psi$, and such that $G \times{ }_{L} L_{\mathfrak{l}}$ is conjugate to $G_{\mathfrak{l}}$ over $L_{\mathfrak{l}}$ for any $\mathfrak{l} \in \mathfrak{L}$.

Let $\mathcal{R}=\left\{\rho_{\lambda}\right\}_{\lambda \in \Lambda}$ be a compatible system of rank $n$ representations of $\Gamma$ with coefficients in $E$. We introduce the following definition.

Definition 4.2 We say that $\mathcal{R}$ has a Lie-irreducible decomposition over $E$ and a subset $\mathcal{L}^{\prime}$ of $\mathcal{L}$ if there exist open subgroups $\Gamma_{i}$ of $\Gamma$, compatible systems $\mathcal{S}_{i}=$ $\left\{\sigma_{i, \lambda}\right\}_{\lambda \in \Lambda^{\prime}}$ of rank $m_{i}$ absolutely Lie-irreducible representations of $\Gamma_{i}$ with coefficients in $E$, where $\Lambda^{\prime}=\cup_{\ell \in \mathcal{L}^{\prime}}|E|_{\ell}$, and rank $d_{i}$ Artin representations ${ }^{3} \omega_{i}$ of $\Gamma_{i}$, for $i=$ $1, \ldots, k$, such that

$$
\rho_{\lambda} \cong \oplus_{i=1}^{k} \operatorname{Ind}_{\Gamma_{i}}^{\Gamma}\left(\sigma_{i, \lambda} \otimes \omega_{i}\right)
$$

over $E_{\lambda}$ for all $\ell \in \mathcal{L}^{\prime}$ and $\lambda \mid \ell$. In this case, we write

$$
\mathcal{R} \cong{ }_{\left(\mathcal{L}^{\prime}\right)} \oplus_{i=1}^{k} \operatorname{Ind}_{\Gamma_{i}}^{\Gamma}\left(\mathcal{S}_{i} \otimes \omega_{i}\right) .
$$

Remark 4.3 For some choices of the group $\Gamma$, one can prove that any $\lambda$-adic representation $\rho: \Gamma \rightarrow \operatorname{GL}_{n}\left(\bar{E}_{\lambda}\right)$ has a Lie-irreducible decomposition $\rho \cong \oplus_{i=1}^{k} \operatorname{Ind}_{\Gamma_{i}}^{\Gamma}\left(\sigma_{i} \otimes \omega_{i}\right)$,

\footnotetext{
2 We say that a rank $n$ representation $\rho$ of $\Gamma$ with coefficients in a field $k$ of characteristic zero is absolutely Lie-irreducible if $\left.\rho\right|_{\Gamma^{\prime}}$ is absolutely irreducible for any open subgroup $\Gamma^{\prime}$ of $\Gamma$.

3 In this setting, we say that a continuous representation $\omega: \Gamma \rightarrow \mathrm{GL}_{d}\left(\bar{E}_{\lambda}\right)$ is Artin if it factors through a finite quotient of $\Gamma$. Any Artin representation $\omega$ can be actually realised over $\overline{\mathbb{Q}}$.
} 
where $\Gamma_{i}$ is an open subgroup of $\Gamma, \sigma_{i}: \Gamma_{i} \rightarrow \mathrm{GL}_{m_{i}}\left(\bar{E}_{\lambda}\right)$ is Lie-irreducible, and $\omega_{i}: \Gamma_{i} \rightarrow \operatorname{GL}_{d_{i}}\left(\bar{E}_{\lambda}\right)$ is Artin, for $i=1, \ldots, k$. This has been proved by Katz, see [12, Proposition 1], when $\Gamma$ is the étale fundamental group of a smooth connected affine curve over an algebraically closed field of positive characteristic, and by Patrikis, see [17, Proposition 3.4.1], by adapting Katz's argument, when $\Gamma$ is the absolute Galois group of a number field. To prove a decomposition result for a compatible system $\mathcal{R}=\left\{\rho_{\lambda}\right\}_{\lambda \in \Lambda}$ of representations of $\Gamma$, one would then start from a single $\lambda$-adic representation $\rho_{\lambda}$ in $\mathcal{R}$, decompose it as $\rho_{\lambda} \cong \bigoplus_{i=1}^{k} \operatorname{Ind}_{\Gamma_{i}}^{\Gamma}\left(\sigma_{i, \lambda} \otimes \omega_{i}\right)$, and extend each $\sigma_{i, \lambda}$ to a compatible system $\mathcal{S}_{i}$ of Lie-irreducible representations. The isomorphism $\mathcal{R} \cong{ }_{\left(\mathcal{L}^{\prime}\right)} \oplus_{i=1}^{k} \operatorname{Ind}_{\Gamma_{i}}^{\Gamma}\left(\mathcal{S}_{i} \otimes \omega_{i}\right)$ would then follow by considerations on the traces of Frobenius elements, see the proofs of Theorem 5.4 and Theorem 6.2. However, extending a $\lambda$-adic representation to a compatible system is a highly non trivial problem, and can be achieved, for instance, under suitable assumptions in the number field case by means of potential automorphy techniques, see Sect. 5, and in the positive characteristic case by means of the global Langlands correspondence for $\mathrm{GL}_{n}$ of L. Lafforgue, see Sect. 6.

Proposition 4.1 has the following consequence.

Corollary 4.4 Let $\mathcal{R}=\left\{\rho_{\lambda}\right\}_{\lambda \in \Lambda}$ be a compatible system of rank $n$ representations of $\Gamma$ with coefficients in $E$. Assume that $\mathcal{R}$ has a Lie-irreducible decomposition over a finite extension $E^{\prime}$ of $E$ and at a subset $\mathcal{L}^{\prime}$ of $\mathcal{L}$. Let $\mathcal{L}^{\prime \prime}$ be a subset of $\mathcal{L}^{\prime}$ such that for each $\ell \in \mathcal{L}^{\prime \prime}$ and $\lambda \mid \ell$ the group $G_{\lambda}^{\circ}$ is unramified. Then, there exist a finite extension $L$ of $E^{\prime}$ and a split connected reductive algebraic subgroup $G$ of $\mathrm{GL}_{n, L}$ defined over $L$ such that for each $\ell \in \mathcal{L}^{\prime \prime}$, each $\lambda \mid \ell$, and each finite place $\mathfrak{l}$ of $L$ lying above $\lambda$, the group $G_{\lambda}^{\circ} \times{ }_{E_{\lambda}} L_{\mathfrak{l}}$ is conjugate to $G \times{ }_{L} L_{\mathfrak{l}}$ over $L_{\mathfrak{l}}$.

Proof By assumption, there exist open subgroups $\Gamma_{i}$ of $\Gamma$, compatible systems $\mathcal{S}_{i}=\left\{\sigma_{i, \lambda^{\prime}}\right\}_{\lambda^{\prime} \in \Lambda^{\prime}}$ of rank $m_{i}$ absolutely Lie-irreducible representations of $\Gamma_{i}$ with coefficients in $E^{\prime}$, where $\Lambda^{\prime}=\cup_{\ell \in \mathcal{L}^{\prime}}\left|E^{\prime}\right|_{\ell}$, and rank $d_{i}$ Artin representations $\omega_{i}$ of $\Gamma_{i}$, for $i=1, \ldots, k$, such that

$$
\rho_{\lambda} \otimes_{E_{\lambda}} E_{\lambda^{\prime}}^{\prime} \cong \oplus_{i=1}^{k} \operatorname{Ind}_{\Gamma_{i}}^{\Gamma}\left(\sigma_{i, \lambda^{\prime}} \otimes \omega_{i}\right)
$$

over $E_{\lambda^{\prime}}^{\prime}$ for each $\ell \in \mathcal{L}^{\prime}$, each $\lambda \mid \ell$, and each $\lambda^{\prime} \mid \lambda$. The algebraic monodromy group $G_{\lambda} \times_{E_{\lambda}} E_{\lambda^{\prime}}^{\prime}$ of $\rho_{\lambda} \otimes_{E_{\lambda}} E_{\lambda^{\prime}}^{\prime}$ only depends on the algebraic monodromy groups of $\sigma_{i, \lambda^{\prime}}$ and $\omega_{i}$, for $i=1, \ldots, k$.

For any $i$ and any $\lambda^{\prime} \in \Lambda^{\prime}$, denote by $H_{\sigma_{i, \lambda^{\prime}}}$ the algebraic monodromy group of $\sigma_{i, \lambda^{\prime}}$. Note that for each $\ell \in \mathcal{L}^{\prime \prime}$ and $\lambda^{\prime} \mid \ell$, the group $H_{\sigma_{i, \lambda^{\prime}}}^{\circ}$ is unramified. Then, by Proposition 4.1, for any $i$ there exist a finite extension $L_{i}$ of $E^{\prime}$ and a connected reductive algebraic subgroup $H_{i}$ of $\mathrm{GL}_{m_{i}, L_{i}}$ defined over $L_{i}$ such that for each $\ell \in \mathcal{L}^{\prime \prime}$, each $\lambda^{\prime} \mid \ell$, and each finite place $\mathfrak{l}$ of $L_{i}$ lying above $\lambda$, the group $H_{\sigma_{i, \lambda^{\prime}}}^{\circ} \times_{E_{\lambda^{\prime}}^{\prime}} L_{i, \mathfrak{l}}$ is conjugate to $H_{i} \times{ }_{L_{i}} L_{i, \mathfrak{l}}$ over $L_{i, \mathfrak{l}}$.

Since for any $i$ the representation $\omega_{i}$ is Artin, it can be defined over $\overline{\mathbb{Q}}$. Its algebraic monodromy group $H_{\omega_{i}}$ is then a finite subgroup of $\mathrm{GL}_{n, \overline{\mathbb{Q}}}$, which can be defined over a number field $L_{\omega_{i}}$. Let us enlarge $L_{i}$ so that it contains $L_{\omega_{i}}$. 
The conclusion follows by taking $L$ to be the composite of the extensions $L_{i}$ for $i=1, \ldots, k$.

\section{Geometric compatible systems of Galois representations}

We see now an application of the abstract results of Sect. 4 to the case of geometric compatible systems of Galois representations attached to automorphic representations. In this context, we refer to [1] for the background terminology.

Let $F$ be a number field, let $\Gamma_{F}$ denote the absolute Galois group of $F$, and let $S$ be a finite set of places of $F$. Let $E$ be a number field, and let $\Lambda$ be a set of finite places of $E$. Let $n \geq 1$ be an integer. We recall the following definition.

Definition 5.1 A geometric ${ }^{4}$ compatible system of rank $n$ representations of $\Gamma_{F}$ defined over $E$ and unramified outside $S$ is a family $\mathcal{R}=\left\{\rho_{\lambda}\right\}_{\lambda \in \Lambda}$ of continuous semisimple representations

$$
\rho_{\lambda}: \Gamma_{F} \rightarrow \mathrm{GL}_{n}\left(\bar{E}_{\lambda}\right)
$$

such that:

(1) If $v \notin S$ is a finite place of $F$, then for all $\lambda$ not dividing the residue characteristic of $v$, the representation $\rho_{\lambda}$ is unramified at $v$, and the characteristic polynomial of $\rho_{\lambda}\left(\right.$ Frob $\left._{v}\right)$ has coefficients in $E$ and is independent of $\lambda$.

(2) Each representation $\rho_{\lambda}$ is de Rham at all places above the residue characteristic of $\lambda$, and in fact crystalline at any place $v \notin S$ above the residue characteristic of $\lambda$.

(3) For each embedding $\tau: F \rightarrow \bar{E}$ the $\tau$-Hodge-Tate weights of $\rho_{\lambda}$ are independent of $\lambda$.

Clearly, any geometric compatible system $\mathcal{R}$ is a compatible system in the sense of Definition 1.1, with $\mathcal{X}=\{(v, \lambda) \in|F| \times \Lambda: v \notin S, \lambda \nmid \operatorname{char}(k(v))\}$, where $k(v)$ denotes the residue field of $F$ at $v$. All the definitions introduced in the case of general compatible systems, as well as all the results proved, are therefore valid also for geometric compatible systems of Galois representations.

Let $F$ be a CM field, let $F^{+}$be the maximal totally real subfield of $F$, and let $c$ be a generator of $\operatorname{Gal}\left(F / F^{+}\right)$. Thanks to the work of many people, e.g. [6,7,11,13,21], geometric compatible systems can be attached to regular algebraic, cuspidal, polarised automorphic representations of $\mathrm{GL}_{n}\left(\mathbb{A}_{F}\right)$. We recall here the general statement of this construction, ${ }^{5}$ which also involves results of [4,22] and [5]. We have:

Theorem 5.2 Let $(\pi, \chi)$ be a regular algebraic, cuspidal, polarised automorphic representation of $\mathrm{GL}_{n}\left(\mathbb{A}_{F}\right)$, unramified outside a finite set $S$ of places of $F$. Then, there

\footnotetext{
4 We add "geometric" to the terminology of $[1, \S 5.1]$ in order to distinguish these compatible systems from the "abstract" compatible systems of Definition 1.1 .

5 To simplify the exposition, in this paper we decided to restrict to the case of imaginary CM fields. Also, an analogous construction holds when $F$ is totally real.
} 
exist a number field $M_{\pi}$, a compatible system $\mathcal{R}_{\pi}=\left\{\rho_{\pi, \lambda}\right\}_{\lambda \in\left|M_{\pi}\right|}$ of rankn semisimple representations

$$
\rho_{\pi, \lambda}: \Gamma_{F} \rightarrow \mathrm{GL}_{n}\left(\bar{M}_{\pi, \lambda}\right)
$$

defined over $M_{\pi}$, and an integer $w$ such that:

(1) $\left(\rho_{\pi, \lambda}, \epsilon_{\lambda}^{1-n} \rho_{\chi, \lambda}\right)$ is a totally odd, polarised $\lambda$-adic representation. ${ }^{6}$

(2) If $v$ is a place of $F$ not dividing the residue characteristic of $\lambda$, then, given an isomorphism $\iota: \bar{M}_{\pi, \lambda} \stackrel{\sim}{\rightarrow} \mathbb{C}$, we have the local-global compatibility

$$
\imath \mathrm{WD}\left(\left.\rho_{\pi, \lambda}\right|_{\Gamma_{F_{v}}}\right)^{\mathrm{F}-\mathrm{ss}} \cong \operatorname{rec}_{v}\left(\pi_{v} \otimes|\operatorname{det}|_{v}^{(1-n) / 2}\right),
$$

and these Weil-Deligne representations are pure of weight $w$.

(3) Each representation $\rho_{\pi, \lambda}$ is de Rham at all places above the residue characteristic of $\lambda$, and for each embedding $\tau: F \rightarrow \bar{M}_{\pi}$ the $\tau$-Hodge-Tate weights of $\rho_{\pi, \lambda}$ have multiplicity at most one, and are given by

$$
\operatorname{HT}_{\tau}\left(\rho_{\pi, \lambda}\right)=\left\{a_{\tau, 1}+n-1, a_{\tau, 2}+n-2, \ldots, a_{\tau, n}\right\},
$$

where $a=\left(a_{\tau, i}\right)$ is the weight of $\pi$. Moreover

$$
\operatorname{HT}_{\tau \circ c}\left(\rho_{\pi, \lambda}\right)=\left\{w-h: h \in \operatorname{HT}_{\tau}\left(\rho_{\pi, \lambda}\right)\right\} .
$$

(4) If $v$ is a place of $F$ dividing the residue characteristic of $\lambda$, then, given an isomorphism $\iota: \bar{M}_{\pi, \lambda} \stackrel{\sim}{\rightarrow} \mathbb{C}$, we have the local-global compatibility

$$
{ }_{\iota} \mathrm{WD}\left(\left.\rho_{\pi, \lambda}\right|_{\Gamma_{F_{v}}}\right)^{\mathrm{F}-\mathrm{ss}} \cong \operatorname{rec}_{v}\left(\pi_{v} \otimes|\operatorname{det}|_{v}^{(1-n) / 2}\right) .
$$

In particular, $\rho_{\pi, \lambda}$ is semistable at $v$, and if $v \notin S$ then it is crystalline.

The compatible system $\mathcal{R}_{\pi}$ is then a geometric compatible system of rank $n$ representations of $\Gamma_{F}$ defined over $M_{\pi}$ and unramified outside $S$, in the sense of Definition 5.1. Moreover, it has coefficients in a finite extension $E_{\pi}$ of $M_{\pi}$ by [6, Proposition 3.2.5], it is totally odd, polarised by the geometric compatible system $\mathcal{M}=\left\{\epsilon_{\lambda}^{1-n} \rho_{\chi, \lambda}\right\}_{\lambda \in\left|M_{\pi}\right|}$ of characters of $\Gamma_{F^{+}}$, and it is strictly pure of weight $w$, and regular.

The global Langlands conjectures in this case predict all the representations $\rho_{\pi, \lambda}$ to be irreducible. For the purposes of this paper, we consider the following weaker conjecture.

Conjecture 5.3 Let $\pi$ be a regular algebraic, polarisable, cuspidal automorphic representation of $\mathrm{GL}_{n}\left(\mathbb{A}_{F}\right)$. Then, there exists a set of rational primes $\mathcal{L}$ of Dirichlet density 1 such that for all $\ell \in \mathcal{L}$ and $\lambda \mid \ell$ the representation

$$
\rho_{\pi, \lambda}: \Gamma_{F} \rightarrow \mathrm{GL}_{n}\left(\bar{M}_{\pi, \lambda}\right)
$$

\footnotetext{
${ }^{6}$ Here $\epsilon_{\lambda}$ denotes the $\lambda$-adic cyclotomic character of $\Gamma_{F^{+}}$, and $\rho_{\chi, \lambda}$ denotes the $\lambda$-adic character of $\Gamma_{F^{+}}$ attached to $\chi$ by global class field theory.
} 
is irreducible.

The proof of this conjecture is a widely open problem in general, and only some partial progress has been obtained so far, see for instance [1, Theorem 5.5.2]. A weaker irreducibility result has been proved in [18]. First of all, let us note that we may assume that the field $M_{\pi}$ is a CM full field of definition for $\mathcal{R}_{\pi}$, see [18, Lemma 1.2, 1.4]. We say that a place $\lambda$ of a CM field $M$ is conjugation invariant if ${ }^{c} \lambda=\lambda$. By [18, Theorem 1.7], there exists a set of rational primes $\mathcal{L}$ of Dirichlet density 1 such that for all $\ell \in \mathcal{L}$ and all conjugation invariant $\lambda \mid \ell$ the representation $\rho_{\pi, \lambda}$ is irreducible. In particular, there exists a set of rational primes $\mathcal{L}^{\prime}$ of positive Dirichlet density such that if $\lambda$ divides some $\ell \in \mathcal{L}^{\prime}$, then $\rho_{\pi, \lambda}$ is irreducible.

Let $F$ be a CM field, let $E$ be a number field, let $\mathcal{L}$ be a set of rational primes of Dirichlet density 1 , and let $\Lambda=\cup_{\ell \in \mathcal{L}}|E|_{\ell}$. We prove the following result.

Theorem 5.4 Let $\mathcal{R}=\left\{\rho_{\lambda}\right\}_{\lambda \in \Lambda}$ be a pure, regular, totally odd, polarisable geometric compatible system of rank $n$ representations of $\Gamma_{F}$ with coefficients in $E$. Then, $\mathcal{R}$ has a Lie-irreducible decomposition over a finite extension $E^{\prime}$ of $E$ and at a subset $\mathcal{L}^{\prime}$ of $\mathcal{L}$ of positive Dirichlet density. Moreover, assuming Conjecture 5.3, we can choose the set $\mathcal{L}^{\prime}$ to have Dirichlet density 1.

Proof By [18, Lemma 1.2, 1.4], there exists a CM full field of definition $M$ for $\mathcal{R}$. Up to enlarging $E$, we can assume that $M \subset E$. Let $\mathcal{M}=\left\{\mu_{\lambda}\right\}_{\lambda \in \Lambda}$ be a geometric compatible system of characters of $\Gamma_{F^{+}}$such that for each $\lambda \in \Lambda$ the pair $\left(\rho_{\pi, \lambda}, \mu_{\lambda}\right)$ is a totally odd, polarised $\lambda$-adic representation. For each $\lambda \in \Lambda$, let

$$
\rho_{\lambda} \otimes_{E_{\lambda}} \bar{E}_{\lambda} \cong \oplus_{i=1}^{k_{\lambda}} \rho_{i, \lambda}
$$

be an irreducible decomposition. Then, if the restriction of $\lambda$ to $M$ is conujugation invariant, the pair $\left(\rho_{i, \lambda}, \mu_{\lambda}\right)$ is a totally odd, polarised $\lambda$-adic representation for each $i=1, \ldots, k_{\lambda}$ by $[18$, Lemma 1.6]. Up to removing finitely many primes from $\mathcal{L}$, we can also assume that if $\ell \in \mathcal{L}$, and $\lambda \mid \ell$, then

- $\ell \geq 2(n+1)$,

- $\zeta_{\ell} \notin F$,

- $\ell$ is unramified in $F$ and lies below no element of the set of bad places for $\mathcal{R}$,

- the Hodge-Tate weights of $\rho_{\lambda}$ lie in a range of the form [a, $\left.a+\ell-2\right]$.

Note that for each $\ell \in \mathcal{L}$ and $\lambda \mid \ell$, and each $i=1, \ldots, k_{\lambda}$, the set of HodgeTate weights of $\rho_{i, \lambda}$ is a subset of the set of Hodge-Tate weights of $\rho_{\lambda}$, and so it is contained in [a, $a+\ell-2]$. It follows by [1, Lemma 1.4.3] that each $\rho_{i, \lambda}$ is potentially diagonalisable at each place $w$ of $F$ lying above $\ell$. By [1, Proposition 5.3.2], there exists a subset $\mathcal{L}^{0}$ of $\mathcal{L}$ of Dirichlet density 1 such that if $\ell \in \mathcal{L}^{0}$, then $\left.\bar{\rho}_{i, \lambda}\right|_{\Gamma_{F\left(\zeta_{\ell}\right)}}$ is irreducible for every $\lambda \mid \ell$, and $i=1, \ldots, k_{\lambda}$. Let $\mathcal{L}^{\prime}$ be the subset of $\mathcal{L}^{0}$ consisting of the primes $\ell$ such that each place of $M$ above $\ell$ in conjugation invariant. This set has positive Dirichlet density by [18, Lemma 1.5]. Let $\Lambda^{\prime}=\cup_{\ell \in \mathcal{L}^{\prime}}|E|_{\ell}$. For each $\lambda \in \Lambda^{\prime}$ and $i=1, \ldots, k_{\lambda}$, by [1, Theorem 5.5.1] the representation $\rho_{i, \lambda}$ is part of a strictly pure compatible system $\mathcal{R}_{i}=\left\{\rho_{i, \lambda}\right\}_{\lambda \in \Lambda^{\prime}}$, with coefficients in a finite extension $E_{i}$ of $E$. By [18, Theorem 1.7], for each $\lambda \in \Lambda^{\prime}$, each $\rho_{i, \lambda}$ is irreducible, and so $k_{\lambda}$ is 
independent of $\lambda \in \Lambda^{\prime}$. Note that assuming Conjecture 5.3, we can find a subset $\mathcal{L}^{\prime}$ of $\mathcal{L}$ of Dirichlet density 1 such that the same conclusion holds. Set $k=k_{\lambda}$ for $\lambda \in \Lambda^{\prime}$.

Fix $i=1, \ldots, k$, and consider the compatible system $\mathcal{R}_{i}$. For all $\lambda \in \Lambda^{\prime}$, let $G_{i, \lambda}$ be the algebraic monodromy group ${ }^{7}$ of $\rho_{i, \lambda}$, and let $G_{i, \lambda}^{\circ}$ be the connected component of the identity of $G_{i, \lambda}$. By Lemma 2.3 there exists a finite Galois extension $F_{i}^{\prime} / F$ such that the representation $\rho_{i, \lambda}$ induces an isomorphism $\operatorname{Gal}\left(F_{i}^{\prime} / F\right) \stackrel{\sim}{\rightarrow} G_{i, \lambda} / G_{i, \lambda}^{\circ}$ for all $\lambda \in \Lambda^{\prime}$. Up to removing finitely many places from $\mathcal{L}^{\prime}$, we may further assume that if $\ell \in \mathcal{L}^{\prime}$, and $\lambda \mid \ell$, then

- $\zeta_{\ell} \notin F_{i}^{\prime}$,

- $\ell$ is unramified in $F_{i}^{\prime}$ and lies below no element of the set of bad places for $\mathcal{R}_{i}$,

Let $F_{i}^{\prime, \mathrm{cm}}$ denote the maximal CM subfield of $F_{i}^{\prime}$, and $F_{i}^{\prime,+}$ the maximal totally real subfield of $F_{i}^{\prime, \mathrm{cm}}$. Then, by [18, Lemma 1.6], for each irreducible component $s$ of $\left.\rho_{i, \lambda}\right|_{\Gamma_{F_{i}^{\prime}}, \mathrm{cm}}$, the pair $\left(s,\left.\mu_{\lambda}\right|_{\Gamma_{F_{i}^{\prime},+}}\right)$ is a totally odd, polarised $\lambda$-adic representation. Again, note that assuming Conjecture 5.3 this holds true above a set $\mathcal{L}^{\prime}$ of primes of Dirichlet density 1.

By [1, Lemma 5.3.1(2)] each $\rho_{i, \lambda}$ is Lie-multiplicity free. ${ }^{8}$ Therefore, for each $\lambda \in$ $\Lambda^{\prime}$, by [17, Lemma 3.4.6(1)] there exist an intermediate field $F \subset F_{i}^{\lambda} \subset F_{i}^{\prime}$, and a rank $m_{i}^{\lambda}$ Lie-irreducible representation $\sigma_{i}^{\lambda}$ of $\Gamma_{F_{i}^{\lambda}}$ such that we can write $\rho_{i, \lambda} \cong \operatorname{Ind}_{\Gamma_{F_{i}^{\lambda}}}^{\Gamma_{F}} \sigma_{i}^{\lambda}$. We now prove that there exists $\lambda_{0} \in \Lambda^{\prime}$ such that $F_{i}^{\lambda_{0}}$ is a CM field. ${ }^{9}$ Denote by $F_{i}^{\lambda, \mathrm{cm}}$ the maximal CM subfield of $F_{i}^{\lambda}$. For simplicity, let us enlarge $E_{i}$ to contain the maximal CM subfield of $F_{i}^{\prime}$, so that $F_{i}^{\lambda, \mathrm{cm}} \subset E_{i}$ for all $\lambda \in \Lambda^{\prime}$, and let us take its Galois closure. If $F_{i}^{\lambda} \neq F_{i}^{\lambda, \mathrm{cm}}$ for all $\lambda \in \Lambda^{\prime}$, then we can find a place $\lambda_{0} \in \Lambda^{\prime}$ with residue characteristic $\ell_{0}$ such that $\ell_{0}$ splits in $E_{i}$ but not in $F_{i}^{\lambda_{0}}$. Let now $w_{0}$ be a non-split place of $F_{i}^{\lambda_{0}}$ of residue characteristic $\ell_{0}$. Since $F_{i, w_{0}}^{\lambda_{0}}$ does not embed in $E_{i, \lambda_{0}}=\mathbb{Q}_{\ell_{0}}$ by assumption, we can deduce by an argument analogous to that of [17, Lemma 3.4.13] that $\rho_{i, \lambda_{0}}$ is not regular, which is a contradiction. Therefore, we get that $F_{i}^{\lambda_{0}}=F_{i}^{\lambda_{0}, \mathrm{~cm}}$. Also, $\ell_{0} \geq 2\left(m_{i}^{\lambda_{0}}+1\right)$ and $\zeta_{\ell_{0}} \notin F_{i}^{\lambda_{0}}$ by the previous assumptions. For simplicity of notation, we set $F_{i}=F_{i}^{\lambda_{0}}$. Also, let $F_{i}^{+}$be the maximal totally real subfield of $F_{i}$.

The representation $\sigma_{i}^{\lambda_{0}}$ is an irreducible component of $\left.\rho_{i, \lambda_{0}}\right|_{\Gamma_{F_{i}}}$, and so the pair $\left(\sigma_{i}^{\lambda_{0}}, \mu_{\lambda_{0}} \mid \Gamma_{F_{i}^{+}}\right)$is a totally odd, polarised $\lambda_{0}$-adic representation, as $F_{i} \subset F_{i}^{\prime, c m}$, and the set of Hodge-Tate weights of $\sigma_{i}^{\lambda_{0}}$ is a subset of the set of Hodge-Tate weights of $\rho_{i, \lambda_{0}}$. This gives that $\sigma_{i}^{\lambda_{0}}$ has Hodge-Tate weights lying in $\left[a, a+\ell_{0}-2\right]$. Since $\ell_{0}$ is unramified in $F_{i}^{\prime}$, and so it is in $F_{i}$ as well, we have that $\sigma_{i}^{\lambda_{0}}$ is crystalline at each

\footnotetext{
7 This is a reductive algebraic subgroup of $\mathrm{GL}_{n, E_{i, \lambda^{\prime}}}$, for $\lambda^{\prime}$ a place of $E_{i}$ above $\lambda$.

8 We say that a rank $n$ representation $\rho$ of a profinite group $\Gamma$ with coefficients in an algebraically closed field $k$ of characteristic zero is Lie-multiplicity free if for any open subgroup $\Gamma^{\prime}$ of $\Gamma$ any irreducible $\Gamma^{\prime}$-subrepresentation of $\rho$ has multiplicity 1 .

9 The following argument is largely adapted on that of [17, Lemma 3.4.13].
} 
place $w_{0}$ of $F_{i}$ lying above $\ell_{0}$ by [17, Lemma 2.2.9]. Therefore, $\sigma_{i}^{\lambda_{0}}$ is potentially diagonalisable at each place $w_{0}$ of $F_{i}$ lying above $\ell_{0}$ by [1, Lemma 1.4.3].

By applying the Mackey restriction formula to $\left.\bar{\rho}_{i, \lambda_{0}}\right|_{\Gamma_{F\left(\zeta \ell_{0}\right)}} \cong \operatorname{Res}_{\Gamma_{F\left(\zeta \ell_{0}\right)}^{\Gamma_{F}} \operatorname{Ind}_{\Gamma_{F}} \Gamma_{F}} \bar{\sigma}_{i}^{\lambda_{0}}$, which is irreducible by assumption, we deduce that $\left.\bar{\sigma}_{i}^{\lambda_{0}}\right|_{\Gamma_{F_{i}\left(\zeta_{\ell_{0}}\right)}}$ is irreducible. Therefore, we can apply [1, Theorem 5.5.1] to get that $\sigma_{i}^{\lambda_{0}}$ is part of a strictly pure compatible system $\mathcal{S}_{i}=\left\{\sigma_{i, \lambda}\right\}_{\lambda \in \Lambda^{\prime}}$ of representations of $\Gamma_{F_{i}}$ with coefficients in a finite extension $E_{i}^{\prime}$ of $E_{i}$.

Since $\mathcal{S}_{i}$ is regular, each representation $\sigma_{i, \lambda}$ is Lie-multiplicity free, again by [1, Lemma 5.3.1(2)]. Also, each $\sigma_{i, \lambda}$ is irreducible by [18, Theorem 1.7]. Since $\sigma_{i, \lambda_{0}} \cong$ $\sigma_{i}^{\lambda_{0}}$ is Lie-irreducible, and Lie-irreducibility in a compatible system of irreducible, Lie-multiplicity free representations is independent of $\lambda$ by [17, Corollary 3.4.11], we get that $\sigma_{i, \lambda}$ is Lie-irreducible for each $\lambda \in \Lambda^{\prime}$. As above, let us remark that assuming Conjecture 5.3, we can find a set $\mathcal{L}^{\prime}$ of primes of Dirichlet density 1 containing $\ell_{0}$, and such that this conclusion holds.

For every finite place $v$ of $F$ outside the set of bad places for $\mathcal{R}_{i}$ and every $\lambda \in \Lambda^{\prime}$ not lying above the residue characteristic of $v$ we have that

$$
\begin{aligned}
\operatorname{Tr} \rho_{i, \lambda}\left(\operatorname{Frob}_{v}\right) & =\operatorname{Tr} \rho_{i, \lambda_{0}}\left(\operatorname{Frob}_{v}\right) \\
& =\operatorname{Tr} \operatorname{Ind}_{\Gamma_{F_{i}}}^{\Gamma_{F}} \sigma_{i}^{\lambda_{0}}\left(\operatorname{Frob}_{v}\right) \\
& =\operatorname{Tr} \operatorname{Ind}_{\Gamma_{F_{i}}}^{\Gamma_{F}} \sigma_{i, \lambda}\left(\operatorname{Frob}_{v}\right),
\end{aligned}
$$

where the first equality follows from the independence of $\lambda$ of the characteristic polynomials at the Frobenius elements in the compatible system $\mathcal{R}_{i}$, and the last equality follows from the usual formula for the trace of an induced representation, see $[1, \S 5.5]$ for instance, and the independence of $\lambda$ of the characteristic polynomials at the Frobenius elements in the compatible system $\mathcal{S}_{i}$. Combining the Čebotarev density theorem with [3, §12.1, Proposition 3] we deduce that $\rho_{i, \lambda} \cong \operatorname{Ind}_{\Gamma_{F_{i}}}^{\Gamma_{F}} \sigma_{i, \lambda}$ for every $\lambda \in \Lambda^{\prime}$.

Let $E^{\prime}$ be a finite extension of $E$ containing all the fields $E_{i}^{\prime}$. Descend each $\sigma_{i, \lambda}$ to a representation $\sigma_{i, \lambda^{\prime}}$ over $E_{\lambda^{\prime}}^{\prime}$ for $\lambda^{\prime} \mid \lambda$. Since $\sigma_{i, \lambda^{\prime}}$ is absolutely Lie-irreducible for each $\ell \in \mathcal{L}^{\prime}$ and $\lambda^{\prime} \mid \ell$, we have that

$$
\mathcal{R} \otimes_{E} E^{\prime} \cong_{\left(\mathcal{L}^{\prime}\right)} \oplus_{i=1}^{k} \operatorname{Ind}_{\Gamma_{F_{i}}}^{\Gamma_{F}} \mathcal{S}_{i} \otimes_{E_{i}^{\prime}} E^{\prime}
$$

is a Lie-irreducible decomposition over $E^{\prime}$ and at $\mathcal{L}^{\prime}$.

Combining this with Corollary 4.4 we get the following result.

Corollary 5.5 Let $\mathcal{R}=\left\{\rho_{\lambda}\right\}_{\lambda \in \Lambda}$ be a pure, regular, totally odd, polarisable geometric compatible system of rank $n$ representations of $\Gamma_{F}$ with coefficients in $E$. Then, there exist a subset $\mathcal{L}^{\prime}$ of $\mathcal{L}$ of positice Dirichlet density, a finite extension $L$ of $E$, and a split connected reductive algebraic subgroup $G$ of $\mathrm{GL}_{n, L}$ such that for each $\ell \in \mathcal{L}^{\prime}$, each $\lambda \mid \ell$, and each finite place $\mathfrak{l}$ of $L$ lying above $\lambda$, the group $G_{\lambda}^{\circ} \times E_{\lambda} L_{\mathfrak{l}}$ is conjugate to $G \times{ }_{L} L_{\mathfrak{l}}$ over $L_{\mathfrak{l}}$. Moreover, assuming Conjecture 5.3, we can choose the set $\mathcal{L}^{\prime}$ to have Dirichlet density 1. 
Remark 5.6 Note that if the compatible system $\mathcal{R}$ has extremely regular weights, then the sets $\mathcal{L}^{\prime}$ of Theorem 5.4 and Corollary 5.5 can be chosen to have Dirichlet density 1 unconditionally.

Remark 5.7 Let $\pi$ be a regular algebraic, polarisable, cuspidal automorphic representation of $\mathrm{GL}_{n}\left(\mathbb{A}_{F}\right)$, and let $\mathcal{R}_{\pi}=\left\{\rho_{\pi, \lambda}\right\}_{\lambda \in\left|M_{\pi}\right|}$ be the corresponding compatible system of representations of $\Gamma_{F}$. Then, the conclusions of Theorem 5.4 and Corollary 5.5 clearly hold for $\mathcal{R}_{\pi}$. We then have that there exist a finite extension $E^{\prime}$ of $E_{\pi}$, a set $\mathcal{L}^{\prime}$ of rational primes of positive Dirichlet density, a finite (possibly trivial) CM extension $F_{\pi}$ of $F$, and a compatible system $\mathcal{S}=\left\{\sigma_{\lambda}\right\}_{\lambda \in \Lambda^{\prime}}$ of absolutely Lieirreducible representations of $\Gamma_{F_{\pi}}$ with coefficients in $E^{\prime}$, where $\Lambda^{\prime}=\left.\left.\cup_{\ell \in \mathcal{L}^{\prime}}\right|^{\prime}\right|_{\ell}$, such that $\mathcal{R}_{\pi} \otimes_{E_{\pi}} E^{\prime} \cong \mathcal{L}^{\prime} \operatorname{Ind}_{\Gamma_{F_{\pi}}}^{\Gamma_{F}} \mathcal{S}$. Notice in particular that the compatible system $\mathcal{S}$ is by construction the compatible system attached to a regular algebraic, polarisable, cuspidal automorphic representation $\tau$ of $\mathrm{GL}_{m}\left(\mathbb{A}_{F_{\pi}}\right)$, and so $\pi$ should be thought of as the "automorphic induction" of $\tau$ from $F_{\pi}$. In analogy with the case of modular forms of weight $k \geq 2$, we suggest to think of $\pi$ as having "complex multiplication" by $F_{\pi}$. Also, we have that there exists a connected reductive group $G_{\pi}$ defined over a finite extension of $E_{\pi}$ which interpolates the groups of connected components of the algebraic monodromy groups of $\rho_{\pi, \lambda}$, for $\lambda$ above a set of primes of positive Dirichlet density. In analogy with the Mumford-Tate conjecture, the group $G_{\pi}$ should be related to a notion of "Mumford-Tate group" for $\pi$.

\section{Compatible systems in the positive characteristic case}

In this last section we see applications of the abstract independence results of Sect. 4 to positive characteristic settings. We start with the case of compatible systems of representations of the absolute Galois group of a global function field, and we then move to compatible systems of lisse sheaves on a variety over a finite field.

Let $p$ be a prime, let $q$ be a power of $p$, and let $C$ be a smooth projective curve, geometrically connected over $\mathbb{F}_{q}$. Let $F$ be the field of rational functions on $C$, and let $\Gamma_{F}$ denote the absolute Galois group of $F$. The following preliminary result is essentially a reformulation in the characteristic $p$ setting of [17, Proposition 3.4.1], which in turn adapts the arguments of [12, Proposition 1].

Lemma 6.1 Let $\rho: \Gamma_{F} \rightarrow \mathrm{GL}_{n}\left(\overline{\mathbb{Q}}_{\ell}\right)$ be an irreducible representation. Then either $\rho$ is induced from a representation of an open proper subgroup of $\Gamma_{F}$, or $\rho$ is Lieirreducible, or there exist an integer $d \geq 2$ dividing $n$, a Lie-irreducible representation $\sigma$ of $\Gamma_{F}$ of dimension $n / d$, and an Artin representation $\omega$ of $\Gamma_{F}$ of dimension d such that $\rho \cong \sigma \otimes \omega$.

The proof of this lemma is just the same as the proof of [17, Proposition 3.4.1], where the key ingredient is the vanishing of $H^{2}\left(\Gamma_{F}, \mathbb{Q} / \mathbb{Z}\right)$ given by Tate's theorem. This holds true also in the positive characteristic case, see [20, Theorem 4].

Let $E$ be a number field, let $\mathcal{L}$ be a set of rational primes of Dirichlet density 1, and let $\Lambda=\cup_{\ell \in \mathcal{L}}|E|_{\ell}$. We prove the following result. 
Theorem 6.2 Let $\mathcal{R}=\left\{\rho_{\lambda}\right\}_{\lambda \in \Lambda}$ be a compatible system of semisimple representations of $\Gamma_{F}$ with coefficients in $E$. Then, $\mathcal{R}$ has a Lie-irreducible decomposition over a finite extension $E^{\prime}$ of $E$ and at $\mathcal{L} \backslash\{p\}$.

Proof Fix $\lambda_{0} \in \Lambda_{\neq p}$. By Lemma 6.1 there exist finite extensions $F_{i}$ of $F$, Lieirreducible representations $\sigma_{i}: \Gamma_{F_{i}} \rightarrow \mathrm{GL}_{m_{i}}\left(\bar{E}_{\lambda_{0}}\right)$, Artin representations $\omega_{i}: \Gamma_{F_{i}} \rightarrow$ $\mathrm{GL}_{d_{i}}\left(\bar{E}_{\lambda_{0}}\right)$, for $i=1, \ldots, k$, such that

$$
\rho_{\lambda_{0}} \otimes_{E_{\lambda_{0}}} \bar{E}_{\lambda_{0}} \cong \oplus_{i=1}^{k} \operatorname{Ind}_{\Gamma_{F_{i}}}^{\Gamma_{F}}\left(\sigma_{i} \otimes \omega_{i}\right)
$$

For each $i$, choose $\chi_{i}: \Gamma_{F_{i}} \rightarrow \bar{E}_{\lambda_{0}}^{\times}$such that the determinant of $\tau_{i}=\sigma_{i} \otimes \chi_{i}$ has finite order. Then, $\tau_{i}$ extends to a compatible system $\left\{\tau_{i, \lambda}\right\}_{\lambda \in \Lambda_{\neq p}}$ by the global Langlands correspondence [14, Théorème VI.9]. Also, by global class field theory we can extend $\chi_{i}$ to a compatible system $\left\{\chi_{i, \lambda}\right\}_{\lambda \in \Lambda_{\neq p}}$. For each $\lambda \in \Lambda_{\neq p}$, let $\sigma_{i, \lambda}=$ $\tau_{i, \lambda} \otimes \chi_{i, \lambda}^{-1}$. We then have that $\mathcal{S}_{i}=\left\{\sigma_{i, \lambda}\right\}_{\lambda \in \Lambda_{\neq} \neq}$is a compatible system extending $\sigma_{i}$.

Let $E^{\prime}$ be a finite extension of $E$ such that each $\mathcal{S}_{i}$ has coefficients in $E^{\prime}$, and each $\omega_{i}$ can be realised over $E^{\prime}$. Then, for any place $v$ of $F$ and any $\lambda \in \Lambda_{\neq p}$ not lying above the residue characteristic of $v$ we have

$$
\begin{aligned}
\operatorname{Tr} \rho_{\lambda}\left(\operatorname{Frob}_{v}\right) & =\operatorname{Tr} \rho_{\lambda_{0}}\left(\operatorname{Frob}_{v}\right) \\
& =\operatorname{Tr} \oplus_{i=1}^{k} \operatorname{Ind}_{\Gamma_{F_{i}}}^{\Gamma_{F}}\left(\sigma_{i} \otimes \omega_{i}\right)\left(\operatorname{Frob}_{v}\right) \\
& =\operatorname{Tr} \oplus_{i=1}^{k} \operatorname{Ind}_{\Gamma_{F_{i}}}^{\Gamma_{F}}\left(\sigma_{i, \lambda} \otimes \omega_{i}\right)\left(\operatorname{Frob}_{v}\right),
\end{aligned}
$$

by independence of $\lambda$ of the characteristic polynomials at the Frobenius elements, and by the usual formulas for the traces of direct sums, tensor products, and induced representations. Combining the Čebotarev density theorem with [3, §12.1, Proposition 3], it then follows that

$$
\mathcal{R} \otimes_{E} E^{\prime} \cong_{\mathcal{L} \backslash\{p\}} \oplus_{i=1}^{k} \operatorname{Ind}_{\Gamma_{F_{i}}}^{\Gamma_{F}}\left(\mathcal{S}_{i} \otimes \omega_{i}\right) \otimes_{E} E^{\prime}
$$

is a Lie-irreducible decomposition for $\mathcal{R}$ over $E^{\prime}$.

By Corollary 4.4 we then have the following immediate consequence.

Corollary 6.3 Let $\mathcal{R}=\left\{\rho_{\lambda}\right\}_{\lambda \in \Lambda}$ be a compatible system of semisimple representations of $\Gamma_{F}$ with coefficients in $E$. Then, there exist a subset $\mathcal{L}^{\prime}$ of $\mathcal{L} \backslash\{p\}$ of Dirichlet density 1, a finite extension $L$ of $E$, and a split connected reductive algebraic subgroup $G$ of $\mathrm{GL}_{n, L}$ such that for each $\ell \in \mathcal{L}^{\prime}$, each $\lambda \mid \ell$, and each finite place $\mathfrak{l}$ of $L$ lying above $\lambda$, the group $G_{\lambda}^{\circ} \times E_{\lambda} L_{\mathfrak{l}}$ is conjugate to $G \times{ }_{L} L_{\mathfrak{l}}$ over $L_{\mathfrak{l}}$.

Let us now consider the case of compatible systems of lisse sheaves on a variety over $\mathbb{F}_{q}$. Refer to $[10, \S 1.1-\S 1.3]$ for the basic definitions and results in this setting.

Let $X$ be a geometrically connected variety over $\mathbb{F}_{q}$. Let $\bar{\eta}$ be a geometric point of $X$, and let $\pi_{1}^{\text {ét }}(X, \bar{\eta})$ be the arithmetic fundamental group of $X$. Let $\ell \neq p$ be a prime. 
The functor which assigns to each lisse $\overline{\mathbb{Q}}_{\ell}$-sheaf $\mathcal{F}$ on $X$ its fibre $\mathcal{F}_{\bar{\eta}}$ over $\bar{\eta}$ induces an equivalence between the category of lisse $\overline{\mathbb{Q}}_{\ell}$-sheaves on $X$ and the category of finite dimensional continuous representations of $\pi_{1}^{\text {et }}(X, \bar{\eta})$ with coefficients in $\overline{\mathbb{Q}}_{\ell}$. Via this equivalence, standard notions about representations (e.g semisimplicity, irreducibility, or Lie-irreducibility) can be translated to the context of lisse sheaves.

Let $E$ be a number field, and let $\Lambda$ be a set of finite places of $E$. We recall the following definition.

Definition 6.4 A compatible system of lisse $E_{\lambda}$-sheaves on $X$ is a family $\mathcal{R}=\left\{\mathcal{F}_{\lambda}\right\}_{\lambda \in \Lambda}$ of lisse $E_{\lambda}$-sheaves $\mathcal{F}_{\lambda}$ on $X$ such that, for any closed point $x \in|X|$ of $X$ and any $\lambda \in \Lambda$, the polynomial $\operatorname{det}\left(1-\operatorname{Frob}_{x} t, \mathcal{F}_{\lambda}\right)$ has coefficients in $E$ and is independent of $\lambda$.

Any compatible system of lisse $E_{\lambda}$-sheaves $\mathcal{R}=\left\{\mathcal{F}_{\lambda}\right\}_{\lambda \in \Lambda}$ on $X$ defines a compatible system $\left\{\rho_{\lambda}\right\}_{\lambda \in \Lambda_{\neq p}}$ of representations of $\pi_{1}^{\text {et }}(X, \bar{\eta})$ with coefficients in $E$ and indexed by $\Lambda$, in the sense of Definition 1.1, where $\rho_{\lambda}$ is the representation $\mathcal{F}_{\lambda, \bar{\eta}}$, and $\mathcal{X}=|X| \times \Lambda_{\neq p}$. The algebraic monodromy group $G_{\lambda}$ of $\rho_{\lambda}$ is called the arithmetic monodromy group of $\mathcal{F}_{\lambda}$. As usual, we denote by $G_{\lambda}^{\circ}$ the connected component of the identity of $G_{\lambda}$. Also, for simplicity of notation we omit the base point $\bar{\eta}$ and write just $\pi_{1}^{\text {ét }}(X)$ for the étale fundamental group of $X$.

Assume that $\Lambda=\cup_{\ell \in \mathcal{L}}|E|_{\ell}$, where $\mathcal{L}$ is a set of rational primes of Dirichlet density 1. From Corollary 6.3 we deduce the following $\lambda$-independence result.

Corollary 6.5 Let $X$ be a normal geometrically connected irreducible variety over $\mathbb{F}_{q}$, and let $\mathcal{R}=\left\{\mathcal{F}_{\lambda}\right\}_{\lambda \in \Lambda}$ be a compatible system of semisimple lisse $E_{\lambda}$-sheaves on $X$. Then, there exist a subset $\mathcal{L}^{\prime}$ of $\mathcal{L} \backslash\{p\}$ of Dirichlet density 1, a finite extension $L$ of $E$, and a split connected reductive algebraic subgroup $G$ of $\mathrm{GL}_{n, L}$ such that for each $\ell \in \mathcal{L}^{\prime}$, each $\lambda \mid \ell$, and each finite place $\mathfrak{l}$ of L lying above $\lambda$, the group $G_{\lambda}^{\circ} \times{ }_{E_{\lambda}} L_{\mathfrak{l}}$ is conjugate to $G \times{ }_{L} L_{\mathfrak{l}}$ over $L_{\mathfrak{l}}$.

Proof Let $\left\{\rho_{\lambda}\right\}_{\lambda \in \Lambda_{\neq p}}$ be the compatible system of representations of $\pi_{1}^{\text {ét }}(X)$ corresponding to $\mathcal{R}$. By [2, Corollary 3.33] there exist a finite étale cover $\varphi: X^{\prime} \rightarrow X$, and a curve $C$ embedding into $X^{\prime}$ via some $l$ such that $\rho_{\lambda}\left(\varphi_{*} \pi_{1}^{\text {ét }}\left(X^{\prime}\right)\right)=\rho_{\lambda}\left(\varphi_{*} l_{*} \pi_{1}^{\text {ét }}(C)\right)$, for all $\lambda \in \Lambda_{\neq p}$.

For any $\lambda \in \Lambda_{\neq p}$, let $\rho_{\lambda}^{\prime}=\rho_{\lambda} \circ \varphi_{*}$, and let $G_{\lambda}^{\prime}$ be its algebraic monodromy group. Since the morphism $\varphi$ is finite étale, then $\varphi_{*} \pi_{1}^{\text {ét }}\left(X^{\prime}\right)$ is an open subgroup of $\pi_{1}^{\text {ét }}(X)$, and so $G_{\lambda}^{\prime, \circ}=G_{\lambda}^{\circ}$, since restriction to an open subgroup leaves the connected component of the identity of the algebraic monodromy group of a $\lambda$-adic representation invariant.

Up to birational equivalence, we can assume that $C$ is smooth and projective. Let $F$ be the field of rational functions on $C$, and let $\pi_{C}: \Gamma_{F} \rightarrow \pi_{1}^{\text {ét }}\left(X^{\prime}\right)$ be the composition of the natural surjection of $\Gamma_{F}$ onto $\pi_{1}^{\text {ét }}(C)$ with $l_{*}$.

For any $\lambda \in \Lambda_{\neq p}$, let $\rho_{C, \lambda}=\rho_{\lambda}^{\prime} \circ \pi_{C}$. Note that $\rho_{C, \lambda}\left(\Gamma_{F}\right)=\rho_{\lambda}^{\prime}\left(\pi_{1}^{\text {ét }}\left(X^{\prime}\right)\right)$, so that the algebraic monodromy groups of $\rho_{C, \lambda}$ and $\rho_{\lambda}^{\prime}$ coincide. The family $\left\{\rho_{C, \lambda}\right\}_{\lambda \in \Lambda_{\neq p}}$ is a compatible system of semisimple representations of $\Gamma_{F}$, and then, by Corollary 6.3 there exist a subset $\mathcal{L}^{\prime}$ of $\mathcal{L} \backslash\{p\}$ of Dirichlet density 1 , a finite extension $L$ of $E$, and a split connected reductive algebraic subgroup $G$ of $\mathrm{GL}_{n, L}$ such that for each 
$\ell \in \mathcal{L}^{\prime}$, each $\lambda \mid \ell$, and each finite place $\mathfrak{l}$ of $L$ lying above $\lambda$, the group $G_{\lambda}^{\circ} \times E_{\lambda} L_{\mathfrak{l}}$ is conjugate to $G \times{ }_{L} L_{\mathfrak{l}}$ over $L_{\mathfrak{l}}$.

Remark 6.6 A crucial point in the proof of this result is the reduction to the case of curves, which relies on the work of Böckle, Gajda, and Petersen in [2, §3.3]. A similar $\lambda$-independence result, for all $\lambda \in \Lambda_{\neq p}$, has been obtained with a different approach by D'Addezio, see [9, Theorem 4.3.2], assuming the variety $X$ to be also smooth.

Acknowledgements The author would like to thank his $\mathrm{PhD}$ supervisor Andrew Wiles for introducing him to the study of these problems, and for his constant guidance and encouragement. The author would also like to thank Wojciech Gajda for bringing [2] to his attention, and Laura Capuano, Toby Gee, Minhyong Kim, Giacomo Micheli, Damian Rössler, and Jack Thorne for interesting conversations and useful suggestions. Finally, the author would like to thank the anonymous referee for the very helpful comments and suggestions.

Open Access This article is licensed under a Creative Commons Attribution 4.0 International License, which permits use, sharing, adaptation, distribution and reproduction in any medium or format, as long as you give appropriate credit to the original author(s) and the source, provide a link to the Creative Commons licence, and indicate if changes were made. The images or other third party material in this article are included in the article's Creative Commons licence, unless indicated otherwise in a credit line to the material. If material is not included in the article's Creative Commons licence and your intended use is not permitted by statutory regulation or exceeds the permitted use, you will need to obtain permission directly from the copyright holder. To view a copy of this licence, visit http://creativecommons.org/licenses/by/4.0/.

\section{References}

1. Barnet-Lamb, T., Gee, T., Geraghty, D., Taylor, R.: Potential automorphy and change of weight. Ann. Math. 179, 501-609 (2014)

2. Böckle, G., Gajda, W., Petersen, S.: On the semisimplicity of reductions and adelic openness for $E$ rational compatible systems over global function fields. Trans. Am. Math. Soc. 372, 5621-91 (2019)

3. Bourbaki, N.: Algèbre: Modules et Anneaux Semi-simples, vol. 8. Hermann, Paris (1958)

4. Caraiani, A.: Local-global compatibility and the action of monodromy on nearby cycles. Duke Math. J. 161(12), 2311-2413 (2012)

5. Caraiani, A.: Monodromy and local-global compatibility for $l=p$. Algebra Number Theory 8(7), 1597-1646 (2014)

6. Chenevier, G., Harris, M.: Construction of automorphic Galois representations. II. Camb. J. Math. 1, 53-73 (2013)

7. Clozel, L.: Motifs et Formes Automorphes: Applications du Principe de Fonctorialité. In: Clozel, L., Milne, J.S. (eds), Automophic Forms, Shimura Varieties, and $L$-functions-Volume I, volume 10 of Perspect. Math., chapter 2 (pp. 77-159). Academic Press, San Diego (1990)

8. Conrad, B., Gabber, O., Prasad, G.: Pseudo-reductive Groups -. New Math, vol. 26, 2nd edn. Monogr. Cambridge University Press, Cambridge (2015)

9. D'Addezio, M.: The monodromy groups of lisse sheaves and overconvergent $F$-isocrystals. Selecta Math. 26(3), 1-41 (2020)

10. Deligne, P.: La Conjecture de Weil, II. Publ. Math. Inst. Hautes Études Sci. 52, 137-252 (1980)

11. Harris, M., Taylor, R.: The Geometry and Cohomology of Some Simple Shimura Varieties, volume 151 of Ann. of Math. Stud. Princeton University Press, Princeton and Oxford (2001)

12. Katz, N.M.: On the monodromy groups attached to certain families of exponential sums. Duke Math. J. 54(1), 41-56 (1987)

13. Kottwitz, R.E.: On the $\lambda$-adic representations associated to some simple Shimura varieties. Invent. Math. 108, 653-665 (1992)

14. Lafforgue, L.: Chtoucas de Drinfeld et correspondance de Langlands. Invent. Math. 147(1), 1-241 (2002)

15. Larsen, M., Pink, R.: On $\ell$-independence of algebraic monodromy groups in compatible systems of representations. Invent. Math. 107(3), 603-636 (1992) 
16. Mumford, D.: Families of abelian varieties. Proc. Sympos. Pure Math. 9, 347-51 (1966)

17. Patrikis, S.: Variations on a theorem of Tate. Mem. Am. Math. Soc., 258(1238) (2019)

18. Patrikis, S., Taylor, R.: Automorphy and irreducibility of some $l$-adic representations. Compos. Math. 151(2), 207-229 (2015)

19. Serre, J.-P.: Abelian $l$-Adic Representations and Elliptic Curves. W. A. Benjamin, New York (1968)

20. Serre, J.-P.: Modular forms of weight one and Galois representations. In: Algebraic number fields: $L$ functions and Galois properties (Proc. Sympos., Univ. Durham, Durham, 1975), pp. 193-268, London (1977). Academic Press

21. Shin, S.W.: Galois representations arising from some compact Shimura varieties. Ann. Math. 173(3), 1645-1741 (2011)

22. Taylor, R., Yoshida, T.: Compatibility of local and global Langlands correspondences. J. Am. Math. Soc. 20, 467-493 (2007)

Publisher's Note Springer Nature remains neutral with regard to jurisdictional claims in published maps and institutional affiliations. 